\title{
Adapting coculture in vitro models of the blood-brain barrier for use in cancer research: maintaining an appropriate endothelial monolayer for the assessment of transendothelial migration
}

\author{
Elodie Vandenhaute, Aurore Drolez, Emmanuel Sevin, Fabien Gosselet, Caroline Mysiorek and Marie-Pierre Dehouck
}

\begin{abstract}
Although brain metastases are the most common brain tumors in adults, there are few treatment options in this setting. To colonize the brain, circulating tumor cells must cross the blood-brain barrier (BBB), which is situated within specialized, restrictive microvascular endothelium. Understanding how cancer cells manage to transmigrate through the BBB might enable this process to be prevented. In vitro models are dedicated tools for characterizing the cellular and molecular mechanisms that underlie transendothelial migration process, as long as they accurately mimic the brain endothelium's in vivo characteristics. The objective of the present study was to adapt an existing in vitro model of the human BBB for use in studying cancer cell transmigration. The model is based on the coculture of endothelial cells (ECs, derived from cord blood hematopoietic stem cells) and brain pericytes. To allow the migration of cancer cells into the lower compartment, our model had to be transposed onto inserts with a larger pore size. However, we encountered a problem when culturing ECs on large (3- $\mu \mathrm{m})$-pore inserts: the cells crossed the membrane and formed a non-physiological second layer on the lower face of the insert. Using 3- $\mu \mathrm{m}$-pore inserts (in a 12-well plate format), we report here on a method that enables the maintenance of a single monolayer of ECs on the insert's upper face only. Under these chosen conditions, the ECs exhibited typical BBB properties found in the original model (including restricted paracellular permeability and the expression of continuous tight junctions). This modified in vitro model of the human BBB enabled us to investigate the migratory potential of the MDA-MB-231 cell line (derived from highly metastatic human breast cancer cells). Last, the results obtained were compared with the rate of transmigration through endothelia with no BBB features.

Laboratory Investigation (2016) 96, 588-598; doi:10.1038/labinvest.2016.35; published online 22 February 2016
\end{abstract}

During the metastatic cascade, cancer cells disseminate through the bloodstream and reach distant organs. First, the cells enter the lumen of a blood vessel in a process referred to as 'intravasation'. The circulating cancer cells subsequently leave the circulatory system and enter the stroma of a distant organ or tissue (a process referred to as 'extravasation'), where they may give rise to new tumors. ${ }^{1}$ Hence, cancer cells interact with and cross endothelial cell (EC) monolayers two times during the metastatic cascade. Given the importance and complexity of these migration processes, a number of in vivo ${ }^{2}$ and in vitro ${ }^{3,4}$ assays have been developed to investigate the underlying mechanisms.

In vitro models can be used to study the various steps in cancer metastasis (including transendothelial migration) under controlled conditions. ${ }^{5}$ To be of value, these models should at least mimic the in vivo cellular structures in question. Indeed, the properties of a vascular bed can influence cancer cell invasion; ${ }^{6}$ some beds are vascularized by fenestrated capillaries, whereas others have restrictive microvessels (such as the blood-brain barrier (BBB) in the central nervous system (CNS)). The BBB's particular features clearly influence cancer cell transmigration and must therefore be taken into account when investigating this phenomenon. In the case of CNS metastases (which represent a therapeutic challenge because of the few available treatments and the poor prognosis ${ }^{7}$ ), understanding how cancer cells circumvent the BBB to reach the brain parenchyma may help to prevent the initiation of brain metastases derived from

Laboratoire de la Barrière Hémato-Encéphalique-EA 2465, Faculté des Sciences Jean Perrin, Université d'Artois, LBHE, Lens, France

Correspondence: C Mysiorek, PhD, Laboratoire de la Barrière Hémato-Encéphalique-EA 2465, Faculté des Sciences Jean Perrin, Université d'Artois, Rue Jean SouvrazSP18, Lens 62307, France.

E-mail: caroline.mysiorek@univ-artois.fr

Received 1 September 2015; revised 6 January 2016; accepted 14 January 2016 
metastasizing-prone tumors (such as melanoma and breast, lung, renal, and colorectal carcinomas). ${ }^{8}$

In vitro models can be helpful in characterizing the cellular and molecular mechanisms underlying the migration of cancer cells through the $\mathrm{BBB}$, as long as they faithfully reproduce the barrier's in vivo restrictive properties and cellular structure. The in vitro BBB models that are known to best reproduce in vivo $\mathrm{BBB}$ features are based on the coculture of ECs and BBB-inducing cells (such as glial cells, astrocytes, and brain pericytes). ${ }^{9,10}$ In our recently developed in vitro model of the human BBB, ECs derived from human cord blood hematopoietic stem cells are cultured on the surface of $0.4-\mu \mathrm{m}$-pore inserts, together with bovine brain pericytes in the lower chamber. ${ }^{11}$ After 6 days of coculture, the BBB phenotype (ie, expression of BBB markers and functional tightness, assessed by permeability studies using hydrophilic molecules such as Lucifer yellow (LY; carbohydrazide dilithium salt), sodium fluorescein, or sucrose) is induced in the ECs. ${ }^{11}$ However, studies of transendothelial migration ${ }^{1}$ (where cancer cells cross an endothelial monolayer, mimicking extravasation, or intravasation) require inserts with a larger pore size (at least $3 \mu \mathrm{m}$ ).

With this application in mind, we sought to adapt our recently developed in vitro model of the human $\mathrm{BBB}^{11}$ to inserts with $3 \mu \mathrm{m}$ pores, while maintaining an appropriate cellular structure (a smooth monolayer of differentiated ECs) and a high level of membrane tightness. However, as described previously, ${ }^{12}$ large pores may allow ECs to migrate and grow on the lower face of the inserts. Hence, the presence of a multilayered endothelium means that the final cellular structure is no longer representative of the monolayer situation in vivo. In an effort to maintain both the inductive coculture with brain pericytes and a single endothelial monolayer solely on the upper face of the insert, we tested (i) a variety of insert coatings and (ii) a preceding period of EC culture in the absence of medium in the lower compartment. Last, the successfully modified model was used to investigate transmigration of the MDA-MB-231 cell line (derived from a highly aggressive, metastatic human breast cancer), and the results compared with the rate of migration through endothelia with no BBB features (our ECs derived from human cord blood hematopoietic stem cells and human umbilical vein ECs (HUVECs), in monoculture).

\section{MATERIALS AND METHODS}

\section{Design and Maintenance of the Original In Vitro Model} of the Human BBB

Our in vitro model human BBB was designed and maintained as described previously. ${ }^{11}$ In compliance with French legislation, written, informed consent was obtained for the collection of human umbilical cord blood. The study's objectives and protocol were approved by the French Ministry of Higher Education and Research (reference; $\mathrm{CODECOH}$ DC2011-1321). All experiments were carried out in line with the authorized protocol.
Briefly, CD $34^{+}$hematopoietic stem cells were isolated from human umbilical cord blood and differentiated in vitro into ECs using medium containing vascular endothelial growth factor. ${ }^{11,13}$ At this stage, the cells have a cobblestone-like morphology and express high levels of EC markers, including CD31, vascular endothelial cadherin, and von Willebrand factor. ${ }^{11}$ Next, these CD $34^{+}$-derived ECs were seeded onto Transwell inserts (pore size: $0.4 \mu \mathrm{m}$; ref. 3401; Corning, Corning, NY, USA) coated with Matrigel (ref. 354230; BD Biosciences, Franklin Lakes, NJ, USA) diluted 1:48 in Dulbecco's modified Eagle's medium (DMEM) and cultured in the presence of brain pericytes at the bottom of the wells. The ECs differentiated into BBB ECs after 5 to 6 days. Pericytes were seeded in the morning in 12-well plates (50 000 cells per well) and ECs were seeded on inserts in the afternoon of the same day ( 80000 cells per insert). The model was cultured in the EC medium (ECM-5, composed of ECM basal medium (Sciencell, Carlsbad, CA, USA) supplemented with $5 \%(\mathrm{v} / \mathrm{v})$ heat-inactivated fetal calf serum, $1 \%(\mathrm{v} / \mathrm{v})$ EC growth supplement (Sciencell), and $0.5 \%(\mathrm{v} / \mathrm{v})$ gentamycin) in a humidified $5 \% \mathrm{CO}_{2}$ atmosphere. The medium was renewed every 2 days.

In our initial attempt to transpose the model onto $3-\mu \mathrm{m}$ pore inserts (ref. 3402; Corning), we followed the same protocol (Figure 1).

To verify whether the decrease in permeability observed in this setting (Figure 1c) was effectively because of the presence of ECs on both faces of the filter membrane, we added a control condition where both sides were seeded with ECs. Inserts were flipped upside-down in wells and soaked with complete ECM-5 medium before seeding 60000 ECs per filter (lower face). Two days later, the inserts were flipped again, and the previous protocol was applied (seeding of 50000 pericytes in the morning, coating of the upper surface with diluted Matrigel, seeding of 80000 ECs on the upper face of inserts in the afternoon).

\section{Adapting the Coculture In Vitro Model of the Human BBB to 3- $\mu \mathrm{m}$-Pore Inserts}

Coating tests on 3- $\mu m$-pore inserts

A variety of coatings (laminin (ref. L2020; Sigma), poly-Dlysine (PDL, ref. P7280; Sigma), and laminin+PDL were tested by combining them with the diluted Matrigel coating used on $0.4-\mu \mathrm{m}$-pore inserts: (Figure 2).

For triple coatings (Matrigel+laminin+PDL), the inserts were inverted and coated with PDL and let for at least $4 \mathrm{~h}$ under the hood before rinsing three times with water. The last rinse was discarded; the inserts were put back in the 12-well plate and left to dry for half an hour under the flow of the hood. On the following day, inserts were coated with laminin $\left(1 \mu \mathrm{g} / \mathrm{cm}^{2}\right)$ on the upper face of the membrane, rinsed three times with water, and left to dry for half an hour under the flow of the hood. Last, a second coating of the upper face was performed with Matrigel (dilated 1:48 in DMEM), before seeding ECs. 
a

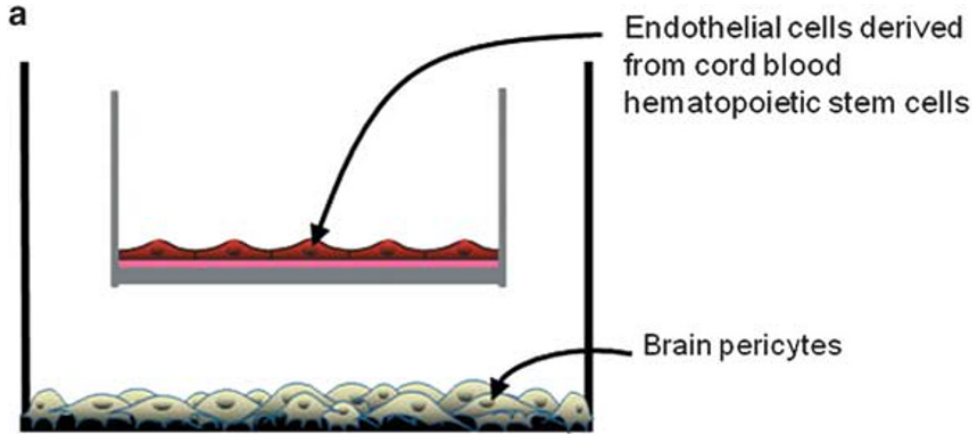

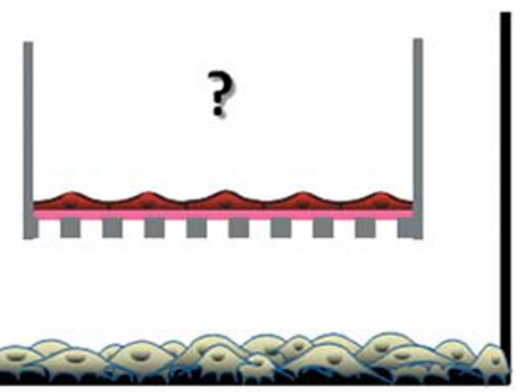

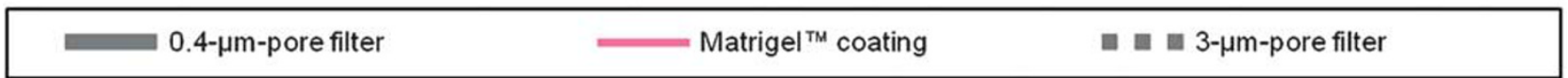

b

\section{Seeding pericytes (wells)}

\section{Coating filters}

3. Seeding endothelial cells (on filters)

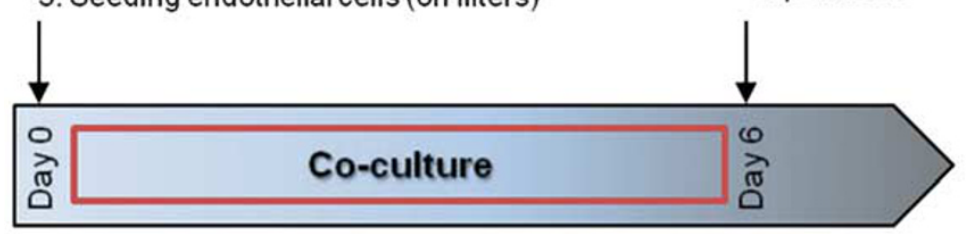

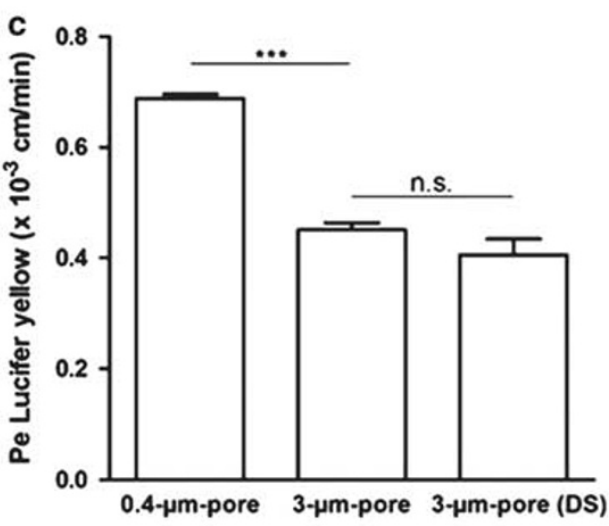

d $0.4-\mu m$-pore filter
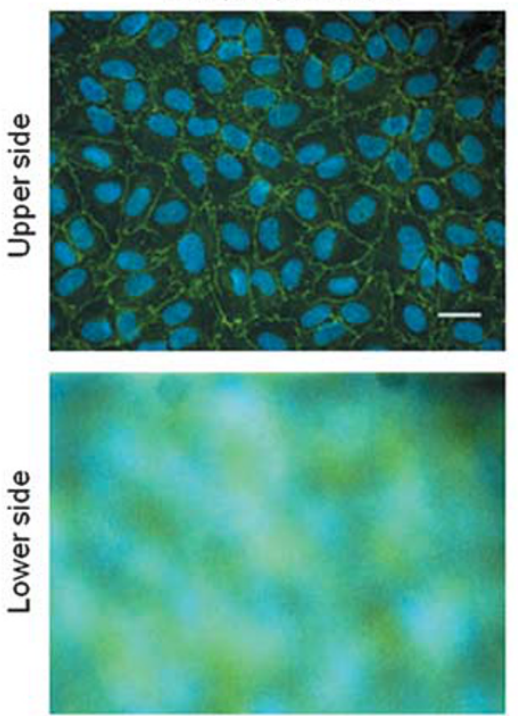

3- $\mu$ m-pore filter
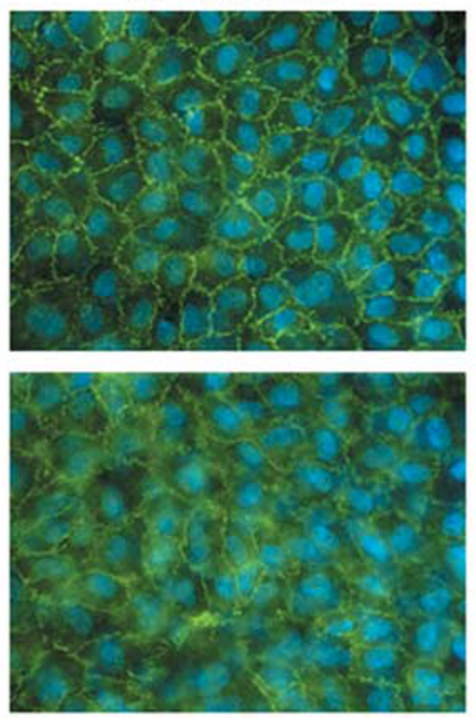

3- $\mu$ m-pore filter (DS)
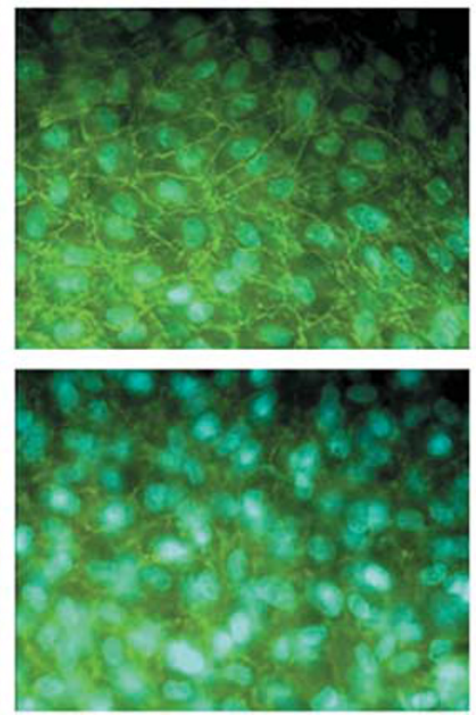

Figure 1 A schematic representation of our original in vitro blood-brain barrier (BBB) model, the protocol for setting it up on $0.4-\mu \mathrm{m}-$ pore inserts, and the features after direct transposition onto $3-\mu \mathrm{m}$-pore inserts. (a) Endothelial cells were derived from cord blood-derived CD34 ${ }^{+}$hematopoietic stem cells. To induce BBB properties, the ECs were cocultured with bovine brain pericytes. ${ }^{11}$ In our original model, ECs were seeded onto $0.4-\mu \mathrm{m}-$ pore inserts (left panel) coated with diluted Matrigel, and pericytes were cultured at the bottom of the wells (right panel). (b) On day 0, pericytes were seeded in 12-well plates. Inserts (0.4- $\mu \mathrm{m}$-pore membranes) were coated with diluted Matrigel and ECs were seeded onto them. Both cell types were then cocultured until day 6 and use of the resulting BBB model in experiments. (c) The permeability (Pe, expressed in $\mathrm{cm} / \mathrm{min}$, on day 6) of the endothelial layer to the low-molecular-weight integrity marker Lucifer yellow (LY) on 0.4- and 3- $\mu \mathrm{m}$-pore inserts. In a third condition, ECs were seeded on each side of the 3- $\mu \mathrm{m}$-pore filter membrane (double seeding condition, DS). The data are quoted as the mean \pm s.e.m. of three experiments, each of which was performed in triplicate. ${ }^{* * *} P<0.001$ in an unpaired $t$-test; n.s., not significant. (d) Representative photographs showing zonula occludens-1 (ZO-1) (in green) and Hoechst (in blue) staining on each face of 0.4 - and $3-\mu \mathrm{m}$-pore inserts. The photographs of the upper and lower faces were taken at exactly the same location on the filter. Scale bar: $25 \mu \mathrm{m}$. 
Concerning the other combinations of coatings, the protocols described before were used to gather laminin and Matrigel, or PDL and Matrigel. The control experiment used Matrigel alone (as in the original model).

\section{Preliminary 'dry-bottom' monoculture tests with 3- $\mu m$-pore inserts}

In this preliminary setting, ECs were seeded (80 000 cells per insert in a 12-well plate format) on inserts maintained with a dry bottom for 1 week ( $500 \mu \mathrm{l}$ of medium in the upper compartment, changed every other day). Next, the inserts were transferred onto pericytes (50 000 cells per well, seeded in 12 -well plates 2 days before the transfer). The resulting coculture was maintained for 5 days - the minimum length of time required for the induction of barrier properties ${ }^{11}$ under standard conditions (a humidified 5\% $\mathrm{CO}_{2}$ atmosphere, with renewal of the ECM-5 medium every 2 days).

\section{Immunohistochemical Staining of Tight Junctions}

ECs were fixed on their inserts using $4 \%(\mathrm{w} / \mathrm{v})$ paraformaldehyde diluted in calcium- and magnesium-free phosphatebuffered saline (PBS-CMF, $10 \mathrm{~min}$ at room temperature (RT)) and permeabilized with Triton X-100 (0.1\% (w/v) in PBS-CMF) for $10 \mathrm{~min}$ at RT. After three rinses with PBS-CMF, the filter membranes were carefully cut out with a scalpel before the following incubation steps. Following a 30-min incubation in PBS-CMF supplemented with 10\% $(\mathrm{v} / \mathrm{v})$ normal goat serum (NGS), cells were incubated for $1 \mathrm{~h}$ at RT with primary antibody against zonula occludens-1 (ZO-1, ref. 617300; Invitrogen; Thermo Fisher Scientific) diluted in PBS-CMF supplemented with 2\% NGS. After three washes in PBS-CMF supplemented with $2 \%$ NGS, the preparations were incubated with secondary antibody for 30 min at RT (goat anti-rabbit Alexa Fluor 568, Invitrogen; dilution: $1 / 200$ in PBS-CMF supplemented with $2 \%$ NGS). Nuclei were stained using Hoechst reagent. Each filter was mounted on a glass slide under a rounded coverslip, using a drop of Mowiol (Sigma-Aldrich) containing 1,4-diazabicyclo [2.2.2] octane (Sigma-Aldrich) as an antiquenching agent. Stained preparations were observed with a fluorescence microscope (DMRD; Leica Microsystems, Wetzlar, Germany). Photographs of the upper and lower sides of the filter were taken at the exact same position. Images were acquired with a high-resolution camera (Cool SNAP RS Photometrics; Leica Microsystems) and processed with Adobe Photoshop software (version 5.5; Adobe Systems, San Jose, CA, USA).

\section{In Vitro Models of Non-Barrier-Forming Peripheral-Like Endothelium: CD34 ${ }^{+}$-Derived ECs and HUVECs Monocultures}

As models of peripheral endothelium with no BBB function, we used $\mathrm{CD}^{2} 4^{+}$-derived ECs ${ }^{11,13}$ and HUVECs (below passage 5). They were cultured in ECM-5 and ECM-2 medium (composed of ECM basal medium supplemented with $2 \%(\mathrm{v} / \mathrm{v})$ heat-inactivated fetal calf serum, $1 \%(\mathrm{v} / \mathrm{v})$ EC growth supplement (Sciencell), and 0.5\% (v/v) gentamycin), respectively. Eighty thousand cells were seeded onto Transwell inserts (pore size: $3 \mu \mathrm{m}$; ref. 3402; Corning) coated with Matrigel diluted 1:48 in DMEM, with no medium in the lower compartment to restrict cells to the upper face of the insert. ${ }^{14}$ Five days later, complete medium was put in the lower compartment. On the following day (after 6 days of culture in total), the endothelial cultures were confluent and the inserts were used in transport and transmigration studies (see the two next paragraphs). For the transmigration experiments, ECM-5 coculture medium was put in the lower compartment to be performed under the exact same conditions as with the in vitro model of the BBB.

\section{Transport Experiments: Permeability of the Endothelium to $\mathbf{L Y}$}

The inserts (in a 12-well format, containing an endothelial layer or merely coated) were transferred into 12-well plates containing $1.5 \mathrm{ml}$ of Ringer-HEPES solution $(150 \mathrm{mM} \mathrm{NaCl}$, $5.2 \mathrm{mM} \mathrm{KCl}, 2.2 \mathrm{mM} \mathrm{CaCl} 2,0.2 \mathrm{mM} \mathrm{MgCl} \cdot 6 \mathrm{H}_{2} \mathrm{O}, 6 \mathrm{mM}$ $\mathrm{NaHCO}_{3}, 5 \mathrm{mM}$ HEPES, $2.8 \mathrm{mM}$ glucose; $\mathrm{pH}$ 7.4) per well (constituting the adluminal compartment). The insert's cell culture medium was removed, and $0.5 \mathrm{ml}$ of Ringer-HEPES solution containing $50 \mu \mathrm{M}$ LY (MW: 457, ref. L0259; Sigma-Aldrich) was added to the upper (luminal) compartment. All incubations were performed at $37^{\circ} \mathrm{C}$. After $60 \mathrm{~min}$, a $200 \mu \mathrm{l}$ aliquot from each lower compartment and a $20 \mu \mathrm{l}$ aliquot from the LY stock solution were placed in a fluorimeter (Synergy H1; BioTek, Winooski, VT, USA) for quantification (excitation wavelength: $425 \mathrm{~nm}$; emission wavelength: $538 \mathrm{~nm}$ ). The endothelial permeability coefficient (Pe) of LY was calculated in $\mathrm{cm} / \mathrm{min}^{15}$ The clearance principle was used to obtain a concentration-independent index of transport. Briefly, the mean volume cleared is plotted $v s$ time, and the slope is estimated by linear regression. The permeability values of the insert (PSf, for inserts with a coating only) and the insert plus endothelium (PSt, for inserts with a coating and cells) were taken into consideration by applying the following equation: $1 / \mathrm{PSe}=1 / \mathrm{PSt}-1 / \mathrm{PSf}$. To obtain the endothelial permeability coefficient $(\mathrm{Pe}$, in $\mathrm{cm} / \mathrm{min}$ ), the permeability value (PSe) corresponding to the endothelium alone was then divided by the insert's porous membrane surface area.

\section{Transmigration Studies}

\section{Culture of the MDA-MB-231 human breast cancer cell line}

MDA-MB-231 human breast cancer cells (ATCC, Manassas, VA, USA) were routinely cultured in DMEM supplemented with $4.5 \mathrm{~g} /$ $1 \mathrm{D}$-glucose, $10 \%(\mathrm{v} / \mathrm{v})$ heat-inactivated fetal calf serum, $1 \%(\mathrm{v} / \mathrm{v})$ L-glutamine and 1\% (v/v) penicillin-streptomycin (ref. 15140122, Gibco; Thermo Fisher Scientific, Waltham, MA, USA).

\section{Transendothelial migration experiment}

MDA-MB-231 cells were labeled with CellTracker Green (Invitrogen), according to the manufacturer's instructions. 
The cells were then treated with EDTA (5 mM in PBS-CMF) before mechanical dissociation in breast cancer cell line medium containing only $1 \%$ of heat-inactivated fetal calf serum. After discarding the medium from cell culture inserts (in the luminal compartment of the in vitro BBB model), breast cancer cells were seeded on $3-\mu \mathrm{m}$-pore filters $\left(4 \times 10^{4}\right.$; $8 \times 10^{4}$ and $16 \times 10^{4}$ cancer cells per filter) containing endothelial monolayers. The following day (after $16 \mathrm{~h}$ of transmigration), inserts were fixed for $10 \mathrm{~min}$ with $4 \%$ paraformaldehyde. After two rinses in PBS-CMF, filters were stained with Hoechst reagent to reveal the cell nuclei. After three final rinsing steps in PBS-CMF, the filter membranes were carefully cut off from the lower face (using a new disposable scalpel (ref. 0503; Swann Morton, Sheffield, UK) for each experiment), and transferred upside-down onto glass slides using tweezers. Between each cutting step, the scalpel and the tweezers were wiped to avoid the accidental transfer of cancer cells from one filter to another. Samples were mounted under rounded coverslips with a drop of Mowiol containing 1,4-diazabicyclo[2.2.2] octane. Stained preparations were observed under the fluorescence microscope (DMRD; Leica Microsystems): for each filter, transmigrated cancer cells (ie, those having crossed the endothelium and the filter membrane into the lower chamber) were counted manually over the entire surface area of the filter membrane (except at the very edge, $\mathrm{x} 40$ objective; wide-field eyepiece). If a filter was not cut and mounted properly, the transmigrated cells were not counted and the experiment repeated. Photographs were acquired with a high-resolution camera (Cool SNAP RS Photometrics; Leica Microsystems).

\section{Migration assay through empty 3- $\mu$ m-pore filters}

The previous protocol was applied to study the migration of MDA-MB-231 cells through empty (ie, containing no endothelial monolayers) 3- $\mu \mathrm{m}$-pore inserts (ref. 3402; Corning) coated with Matrigel (dilution 1/48 in DMEM), with ECM-5 coculture medium in the lower compartment during the migration experiment.

\section{Statistical Analyses}

All data were expressed as the mean \pm s.e.m. from three independent experiments (each performed in triplicate). The threshold for statistical significance (in an unpaired $T$-test) was set to $P<0.05$. All statistical analyses were performed using GraphPad Prism software for Windows (version 5.0;
GraphPad Software, San Diego, CA, USA). Statistical test results are indicated as follows: n.s., not significant; ${ }^{\star} P<0.05$; ${ }^{* *} P<0.01 ;{ }^{* *} P<0.001$.

\section{RESULTS \\ A Layer of ECs on Each Side of 3- $\mu \mathrm{m}$-pore Inserts in a Coculture In Vitro Model of the Human BBB}

Our original in vitro model of the human BBB was set up on 0.4- $\mu$ m-pore inserts ${ }^{11}$ (Figure 1a, left panel): pericytes were seeded in 12-well plates, and ECs derived from cord blood hematopoietic stem cells were seeded on Matrigel-coated inserts on the same day (Figure 1b). The model was ready 6 days later. Under these conditions, the endothelium eventually formed a restrictive barrier, as shown by the low Pe to LY $\left(0.69 \pm 0.01 \times 10^{-3} \mathrm{~cm} / \mathrm{min}\right.$, Figure 1c) and the smooth, continuous aspect of ZO-1 staining at the ECs' borders (Figure 1d, left panels), attesting to the presence of tight junction formation. Hence, this endothelium consisted of a single EC layer on the upper face of the insert membrane (Figure $1 \mathrm{~d}$, left panels), as expected when using $0.4-\mu \mathrm{m}$-pore inserts. $^{11}$

The same protocol (Figure 1b) was used to create a BBB model on 3- $\mu \mathrm{m}$-pore inserts (Figure 1a, right panel). The resulting endothelium was less permeable to LY (Pe: $0.45 \pm 0.01 \times 10^{-3} \mathrm{~cm} / \mathrm{min}$; Figure 1c) than the original model on $0.4-\mu \mathrm{m}$-pore inserts, and expressed continuous tight junctions (Figure 1d, middle panels). However, inspection of the ZO-1 and Hoechst staining patterns revealed the existence of a second monolayer of cells on the lower face of the insert membrane (Figure 1d, middle panels), suggesting that ECs can cross $3-\mu \mathrm{m}$ pores and thus invade the lower face of the membrane. This feature did not appear to be insertdependent because tests on other products (with different materials and/or pore densities) also evidenced extensive migration of ECs to the lower face (data not shown). The migration has also been observed with ECs from other species (ie, the bovine brain capillary ECs extracted and routinely used in our lab; data not shown). This phenomenon is not representative of the BBB's structure in vivo, but it might well explain the lower permeability value observed with $3-\mu \mathrm{m}$ pore inserts (Figure 1c). Indeed, purposely seeding ECs on each face of the insert membrane (double seeding, DS; Figure 1d, right panels) led to a similar decrease in LY permeability (Pe: $0.40 \pm 0.03 \times 10^{-3} \mathrm{~cm} / \mathrm{min}$; Figure 1c).

Figure 2 Testing different coatings to prevent endothelial cells (ECs) from crossing the 3- $\mu$ m-pore insert membrane. (a) Permeability (Pe, expressed in $\mathrm{cm} / \mathrm{min}$ ) to Lucifer yellow (LY) of the endothelial barrier resulting from the use of different combinations of coatings (Matrigel alone, Matrigel+laminin, Matrigel+poly-D-lysine (PDL), and Matrigel+laminin+PDL). LY was used as an integrity marker of the blood-brain barrier (BBB). The protocol was the same as in Figure 1, except that different coatings were used. The results are presented as the mean \pm s.e.m. of three experiments, each of which was performed in triplicate. For statistical analysis, an unpaired $t$-test was performed. n.s., not significant. (b) Representative photographs showing zonula occludens-1 (ZO-1) (in green) and Hoechst (in blue) staining on each face of the insert membrane and in each condition. The photographs of the upper and lower faces were taken at exactly the same location on the filter. Scale bar: $25 \mu \mathrm{m}$. 


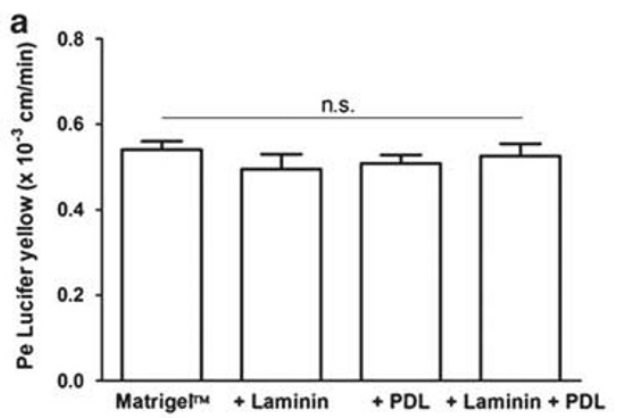

b
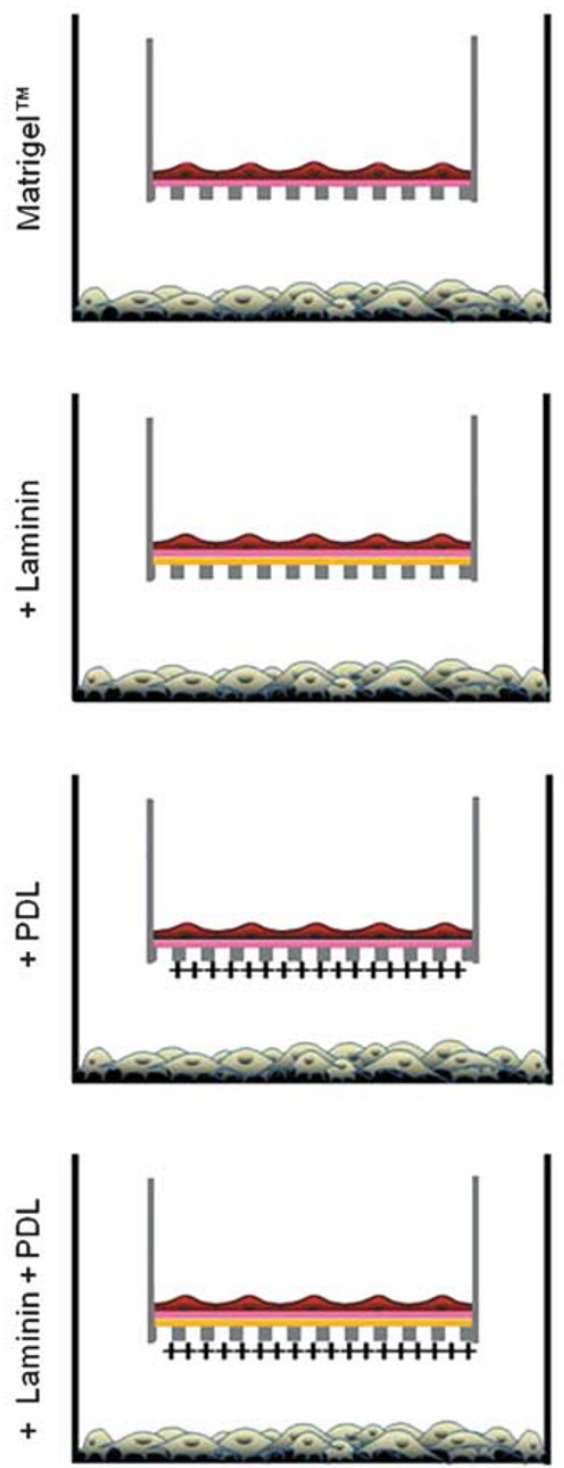

Upper side
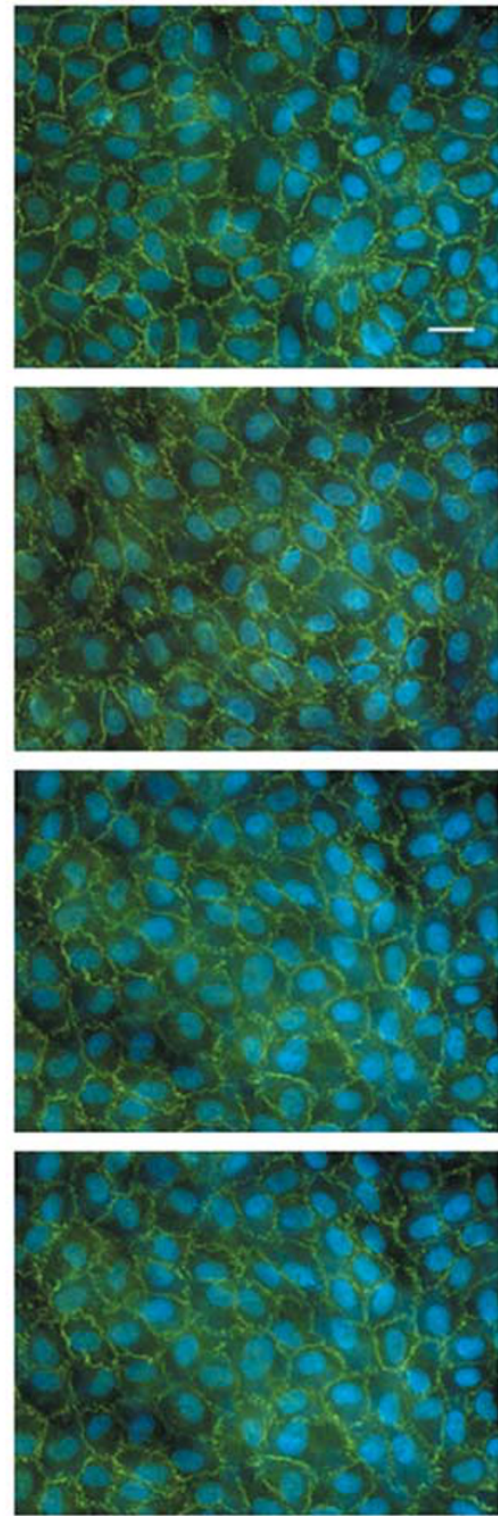

Lower side
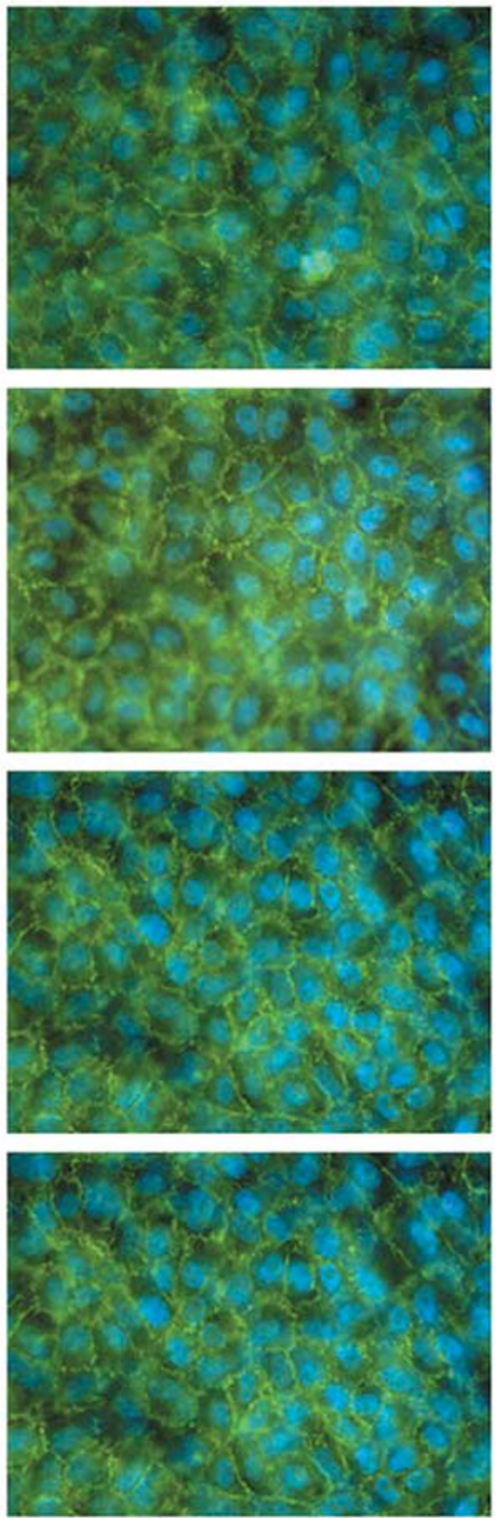
= $= \pm 3-\mu \mathrm{m}$-pore filter
+++ PDL
Laminin
Matrigel $^{\mathrm{TM}}$ 
As the substrate is known to influence EC phenotype, growth and migration in vitro, ${ }^{16,17}$ we tested different combinations of coatings with a view to preventing EC migration to the lower face of the $3-\mu \mathrm{m}$-pore insert membranes.

\section{a}

1. Coating filters

\section{Seeding endothelial cells}

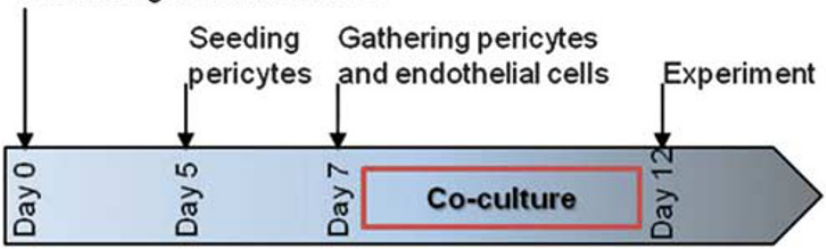

b
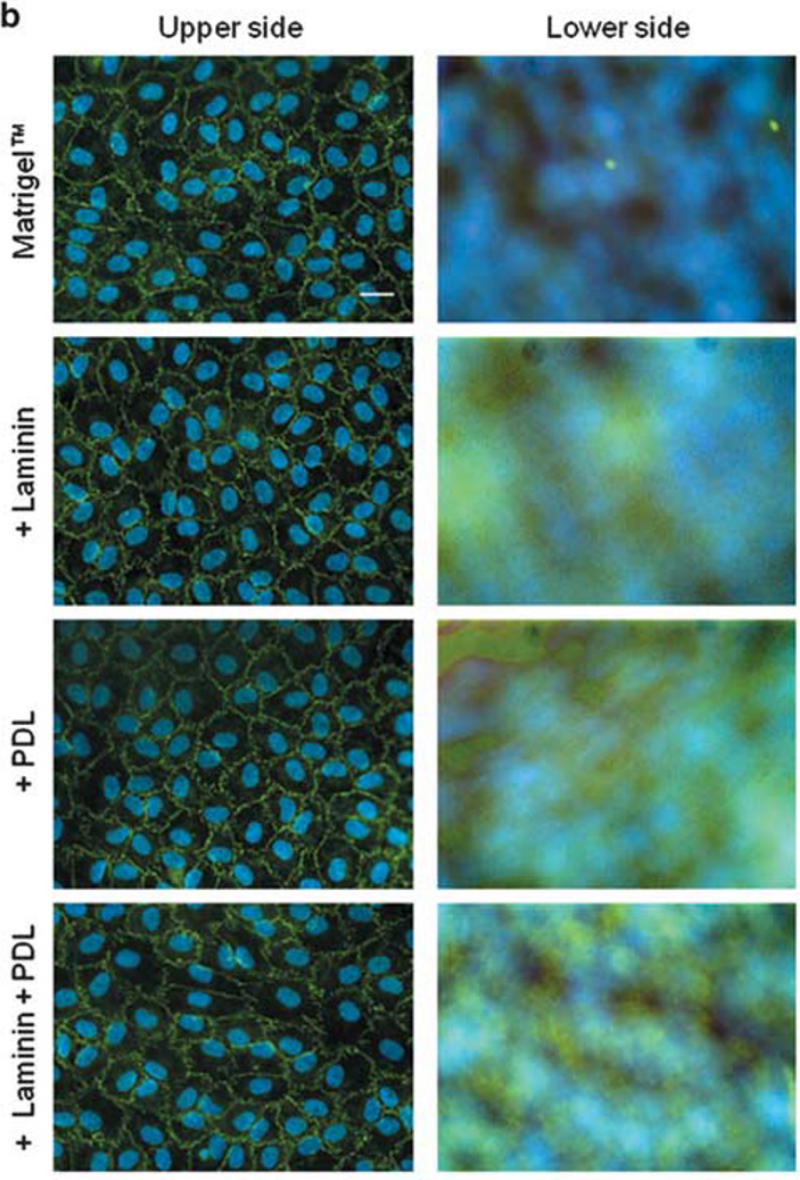

c

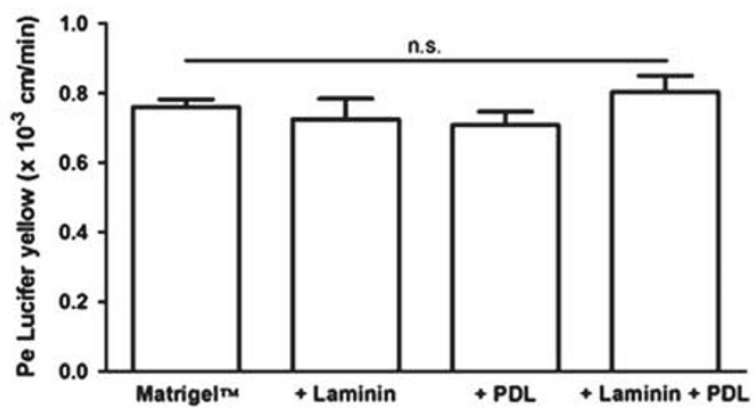

\section{Coating Tests on $\mathbf{3}-\mu \mathrm{m}$-Pore Inserts}

The Matrigel coating used in the original model was combined with laminin and/or PDL on $3-\mu \mathrm{m}$-pore insert coatings (Figure 2). Laminins are expressed in brain capillaries in vivo and are known to provide a good adhesive matrix for brain capillary ECs in vitro, ${ }^{18}$ whereas PDL has been shown to decrease the attachment of primary human corneal ECs in vitro ${ }^{19}$ and was therefore tested as a repulsive component on the lower face of the inserts.

Transport experiments indicated that all the resulting models were poorly permeable to LY (Figure 2a, global mean of $0.52 \pm 0.01 \times 10^{-3} \mathrm{~cm} / \mathrm{min}$ ). Accordingly, the ZO-1 staining pattern was regular and continuous (Figure $2 \mathrm{~b}$ ). However, the various coatings did not prevent ECs from crossing the pores during the coculture, and a second endothelial layer was observed under all conditions tested (Figure 2b).

\section{Prior 'Dry-Bottom' Endothelial Monoculture Tests on 3- $\mu \mathrm{m}$-Pore Inserts}

Although culturing ECs on 'dry-bottom' inserts (ie, with no medium in the lower compartment) restricts cells to the upper face of the insert, ${ }^{14}$ this technique cannot be used with the coculture of cells in the lower well. This idea was implemented by culturing ECs alone with no medium in the lower compartment, before the addition of pericytes to the system. The coatings used in the previous experiments were again tested. After 1 week of monoculture, inserts with ECs were transferred onto wells containing pericytes (Figure 3a). Six days later, the model was used for LY permeability assay and ZO-1 staining. The results indicated the formation of a regular monolayer of ECs (with continuous tight junctions) (Figure 3b, left panel) on the upper face of the insert only. No ECs could be seen on the lower face (Figure 3b, right panel). Whatever the coating tested, the monolayer's permeability was still low (Figure $3 \mathrm{c}$,

Figure 3 Testing a prior 1-week period of endothelial monoculture in the absence of medium in the lower compartment as a means of maintaining ECs on the upper face of the insert only. (a) On day 0, inserts were coated and ECs were seeded as a monoculture, whereas the lower face of the inserts was kept dry (ie, there was no medium in the lower compartment) until day 7. On day 5, pericytes were seeded in 12-well plates (monoculture). Pericytes and ECs were cocultured from day 7 until the day of the experiment (day 12). (b) Representative photographs of zonula occludens-1 (ZO-1) (in green) and Hoechst (in blue) staining on each face of the insert membrane, for each coating combination (Matrigel alone, Matrigel+laminin, Matrigel+poly-D-lysine (PDL), and Matrigel +laminin+PDL), after 7 days of monoculture and then 5 days of coculture. The photographs of the upper and lower faces were taken at exactly the same location on the filter. Scale bar: $25 \mu \mathrm{m}$. (c) Permeability (Pe, in $\mathrm{cm} /$ $\mathrm{min}$ ) of the endothelium to Lucifer yellow (LY) under the different conditions. LY was used as an integrity marker of the blood-brain barrier (BBB). The results are presented as the mean \pm s.e.m. of three experiments, each of which was performed in triplicate. For statistical analysis, an unpaired $t$-test was performed. n.s., not significant. 
overall mean Pe: $0.75 \pm 0.02 \times 10^{-3} \mathrm{~cm} / \mathrm{min}$ ) but was somewhat higher compared with that in the previous experiment (Figure 2a, overall mean Pe: $0.52 \pm 0.01 \times 10^{-3} \mathrm{~cm} / \mathrm{min}$ for a two monolayers of ECs). The original coating (diluted Matrigel only), therefore, appeared to be appropriate for setting up a single-monolayer BBB model with 1 week of monoculture and no medium in the lower compartment. Shorter monoculture periods were tested ( $2-5$ days) but did not give reproducible results (data not shown).

This method was therefore applied to generate a $\mathrm{BBB}$ model with a single monolayer of ECs on the top face of Matrigel-coated 3- $\mu \mathrm{m}$-pore inserts. After 1 week of endothelial monoculture in the absence of medium in the lower compartment, ECs were cocultured with brain pericytes until the day of the transmigration experiment (Figure 3a).

\section{Transmigration of MDA-MB-231 Cells Through the BBB In Vitro}

We quantified the transmigration of CellTracker Greenstained MDA-MB-231 cells through empty 3- $\mu \mathrm{m}$-pore inserts and our modified in vitro model of the human BBB (inserts +BBB-like endothelium) after $16 \mathrm{~h}$ of incubation (Figure 4). As expected, the mean number of cancer cells having reached the lower surface of empty filters increased with the number seeded (Figure 4a): $448.5 \pm 27.5$ migrated cells when 40000 were seeded per filter, $766.0 \pm 45.2$ when 80000 were seeded, and $2426 \pm 180.5$ when 160000 were seeded. The results of the transmigration study through the in vitro $\mathrm{BBB}$ model also demonstrated that the mean number of transmigrated cells rose with the number seeded in the upper compartment: $56.7 \pm 11.5$ transmigrated cells when 40000 were seeded per filter, $105.4 \pm 8.7$ transmigrated cells when 80000 were seeded, and $163.3 \pm 14.0$ when 160000 were seeded (Figures $4 \mathrm{~b}$ and $\mathrm{d}$ ). The number of transmigrated cells was much lower in the presence of the BBB-like endothelium (Figure $4 \mathrm{~b}$ ) compared with that in its absence (Figure 4a), suggesting that the $\mathrm{BBB}$ effectively represents a major hurdle for cancer cell transmigration. Whatever the number of cancer cells seeded in the luminal compartment, the endothelium's paracellular permeability remained stable after $16 \mathrm{~h}$ of contact (relative to control samples, as assessed by LY permeability measurements) (Figure 4c). Observation of the Hoechst staining pattern confirmed that there were still no ECs on the lower face after cancer cell transmigration (Figure 4d). These results suggest that (i) this adapted in vitro $\mathrm{BBB}$ model can be used for transmigration studies and (ii) the transmigration of MDA-MB-231 cells does not damage the integrity of the in vitro BBB model after $16 \mathrm{~h}$.

Interestingly, the rate of transmigration of MDA-MB-231 cells was more important through endothelia with no BBB features (our own CD $34^{+}$-derived endothelium and HUVECs in monoculture; $312.0 \pm 25.7$ and $268.0 \pm 26.5$ when 80000 cancer cells were seeded, respectively) in comparison with the number of transmigrated cells through the in vitro $\mathrm{BBB}$ model (Figure 5a). Monocultures of $\mathrm{CD} 34^{+}$-derived ECs and
HUVECs exhibited a higher permeability to LY (Pe: $1.21 \pm 0.03$ and $1.81 \pm 0.18 \times 10^{-3} \mathrm{~cm} / \mathrm{min}$, respectively) compared with the BBB-like endothelium (Pe: $0.64 \pm 0.03 \times 10^{-3} \mathrm{~cm} / \mathrm{min}$ ). Whatever the model used, LY permeability coefficient was not significantly impacted by the contact with the MDA-MB-231 cells during $16 \mathrm{~h}$ (Figure 5b).

\section{DISCUSSION}

The objective of the present study was to adapt an in vitro BBB model for use in transendothelial migration assays of cancer cells. Indeed, in vitro models that reliably mimic a differentiated human brain microvascular endothelium would be of great value for probing the mechanisms underlying extravasation and brain metastasis initiation. Our coculture model has been characterized on $0.4-\mu \mathrm{m}$-pore inserts; it exhibits features of the BBB such as low permeability to small, hydrophilic molecules, and the expression of continuous tight junctions and specific BBB transporters and receptors. ${ }^{11}$ As with other in vitro BBB models, it combines ECs with the brain microvascular pericytes ${ }^{20-23}$ known to be involved in BBB formation and maintenance in vivo. ${ }^{24,25}$

Unfortunately, when this original model was transposed to $3-\mu \mathrm{m}$-pore inserts, the ECs crossed the porous membrane to form a second layer on the lower face. ${ }^{12}$ In vivo, circulating prometastatic cancer cells pass through a single layer of ECs when they transmigrate through brain microvessels. Hence, the migration of ECs through $3-\mu \mathrm{m}$-pore inserts prevents the model from being used to investigate this process in vitro. This observation of ECs crossing the large pore inserts highlights the need to carefully check in vitro model characteristics before their use in pathophysiological studies in which the three-dimensional cellular structure is important (such as transmigration assays). The presence of another endothelial layer on the lower face of the filter also raises doubts regarding the polarity of the barrier system.

We therefore had to adapt and optimize our original in vitro $\mathrm{BBB}$ model for use with large-pore-sized inserts in transmigration studies, by preventing ECs from forming a second monolayer on the lower face of the insert. Merely changing the insert's coating was not successful in preventing EC migration. In contrast, 7 days of EC monoculture in the absence of medium in the lower compartment were enough to maintain a single monolayer during the subsequent coculture period, whatever the coating used. The resulting endothelial monolayer presented a low Pe value for LY and displayed continuous tight junctions (as in the original model). The solution we adopted therefore consisted in culturing the ECs alone on Matrigel-coated 3- $\mu \mathrm{m}$-pore inserts with no medium in the lower compartment for 7 days, before coculturing them with brain pericytes for 6 days (total time of culture: 13 days). The fact that the prior 'dry-bottom' culture period worked with all combinations of coatings suggests that the mechanism is rather physical than biochemical. This possibility is also suggested by the fact that, each time the lower face of an insert had been accidentally wet with some 

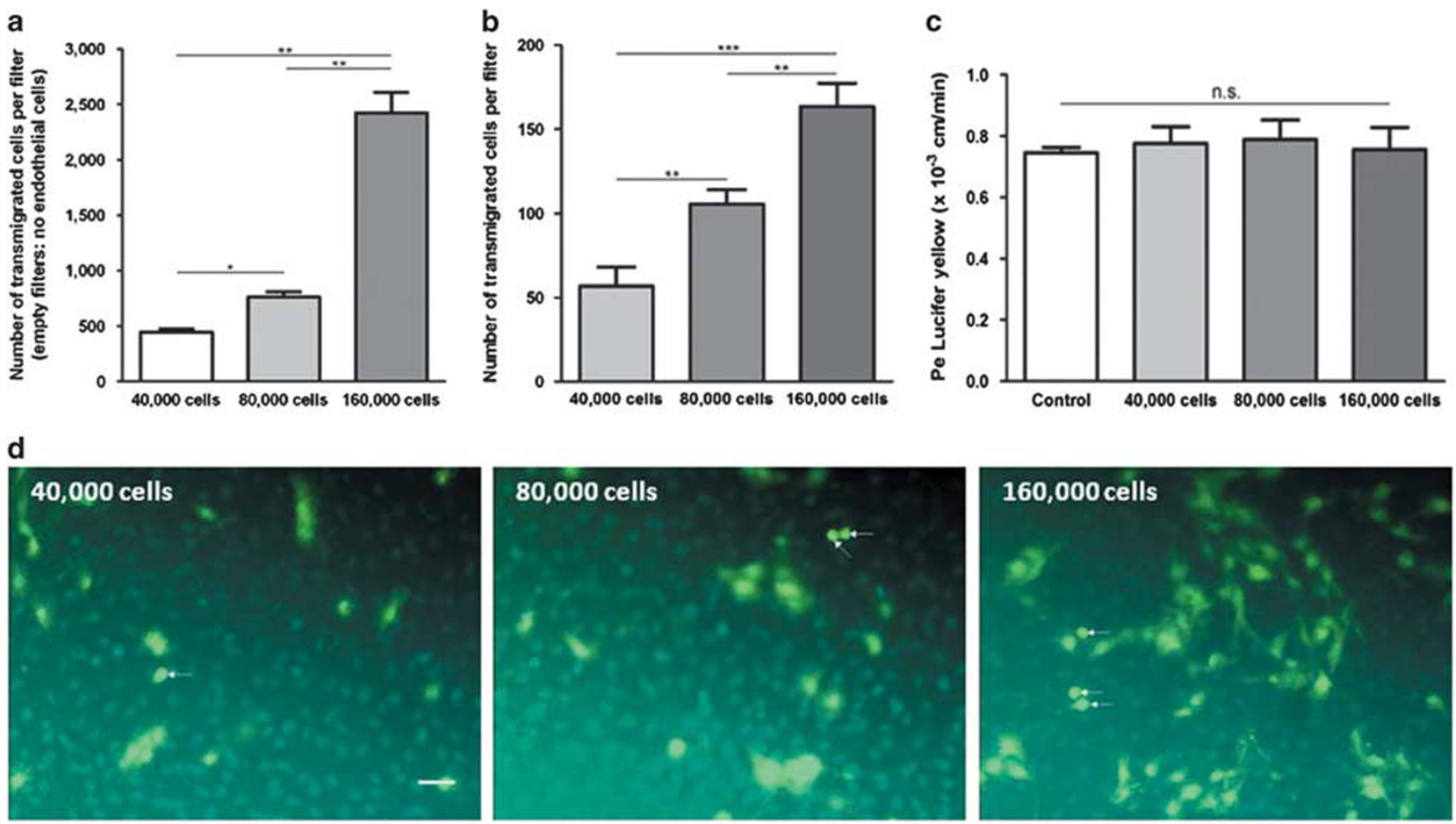

Figure 4 Transmigration of the MDA-MB-231 breast cancer cell line through empty 3- $\mu$ m-pore filters and our in vitro model of the human blood-brain barrier (BBB) (on 3- $\mu \mathrm{m}$-pore inserts). (a) The number of transmigrated MDA-MB-231 cells on the lower face of empty 3- $\mu \mathrm{m}$-pore cell culture inserts after $16 \mathrm{~h}$, as a function of the initial number of cancer cells seeded in the upper compartment. The results are presented as the mean \pm s.e.m. of three experiments, each of which was performed in triplicate. For statistical analysis, an unpaired $t$-test was performed $\left({ }^{*} P<0.05 ;{ }^{*} P<0.01\right)$. (b) The number of transmigrated MDA-MB-231 cells, though our in vitro model of the human BBB after $16 \mathrm{~h}$, as a function of the initial number of cancer cells seeded in the upper compartment. The results are presented as the mean \pm s.e.m. of three experiments, each of which was performed in triplicate. For statistical analysis, an unpaired $t$-test was performed $\left({ }^{* *} P<0.01 ;{ }^{* * *} P<0.001\right)$. (c) Permeability of the endothelium to Lucifer yellow (LY) after a 16-h transmigration experiment, as a function of the initial number of cells seeded in the upper compartment. The results are presented as the mean \pm s.e.m. of three experiments, each of which was performed in triplicate. For statistical analysis, an unpaired $t$-test was performed (n.s., not significant). (d) Representative photographs of CellTracker Green-stained MDA-MB-231 cells (in green) and Hoechst (in blue) staining on the upper and lower face of 3- $\mu \mathrm{m}-$ pore inserts, after $16 \mathrm{~h}$ of transmigration through the BBB. Arrows indicate transmigrated cancer cells. Scale bar: $20 \mu \mathrm{m}$.

medium during the 7-day 'dry-bottom' culture period, ECs invaded the lower face of the inserts before the end of the coculture period (data not shown, personal observation).

A trivial solution would have been to culture the ECs alone on the inserts (with no medium in the lower compartment and no coculture with pericytes) for the complete cell culture process before to the cancer transmigration experiments. ${ }^{14}$ However, it is well established that the microvascular ECs' BBB properties are induced specifically by the brain microenvironment. For this reason, reliable in vitro models require coculture of ECs and glial cells and/or brain pericytes. ${ }^{10}$ Some studies have used medium from glial cell or brain pericyte cultures to obtain a restrictive phenotype in ECs. However, there are probably many different BBB-inducing factors and cell-cell interactions, ${ }^{25,26}$ not all of which have yet been fully characterized. ${ }^{27}$ We therefore preferred to maintain the coculture feature that reliably reproduces the $\mathrm{BBB}$ phenotype ${ }^{11}$ and therefore worked to adapt our original coculture model to use with large-pore-size inserts.
Several cancers are prone to develop brain metastases, including breast cancer. Brain metastases occur in at least $20-30 \%$ of breast cancer patients and thus represent a major cause of cancer morbidity and mortality. ${ }^{28}$ Of the various subtypes of breast cancer, triple-negative cancers (those that do not express the estrogen receptor, the progesterone receptor, and the epidermal growth factor receptor) are associated with an especially high incidence of brain metastasis. ${ }^{29}$ The MDA-MB-231 cell line used in the present study is a triple-negative subtype. We found that (i) a proportion of the MDA-MB-231 cells was able to cross the human in vitro $\mathrm{BBB}$ model within $16 \mathrm{~h}$ and (ii) the interactions and transmigration processes did not cause the BBB to break down (as evidenced by permeability assays with a low-molecular-weight integrity marker). It has been shown that MDA-MB-231 cells can successfully transmigrate through human brain microvascular ECs in vitro. ${ }^{30}$ Application of this syngeneic approach (ie, human cancer cells and human brain-like ECs) to BBB transmigration studies may provide a better understanding of the initial cellular and 

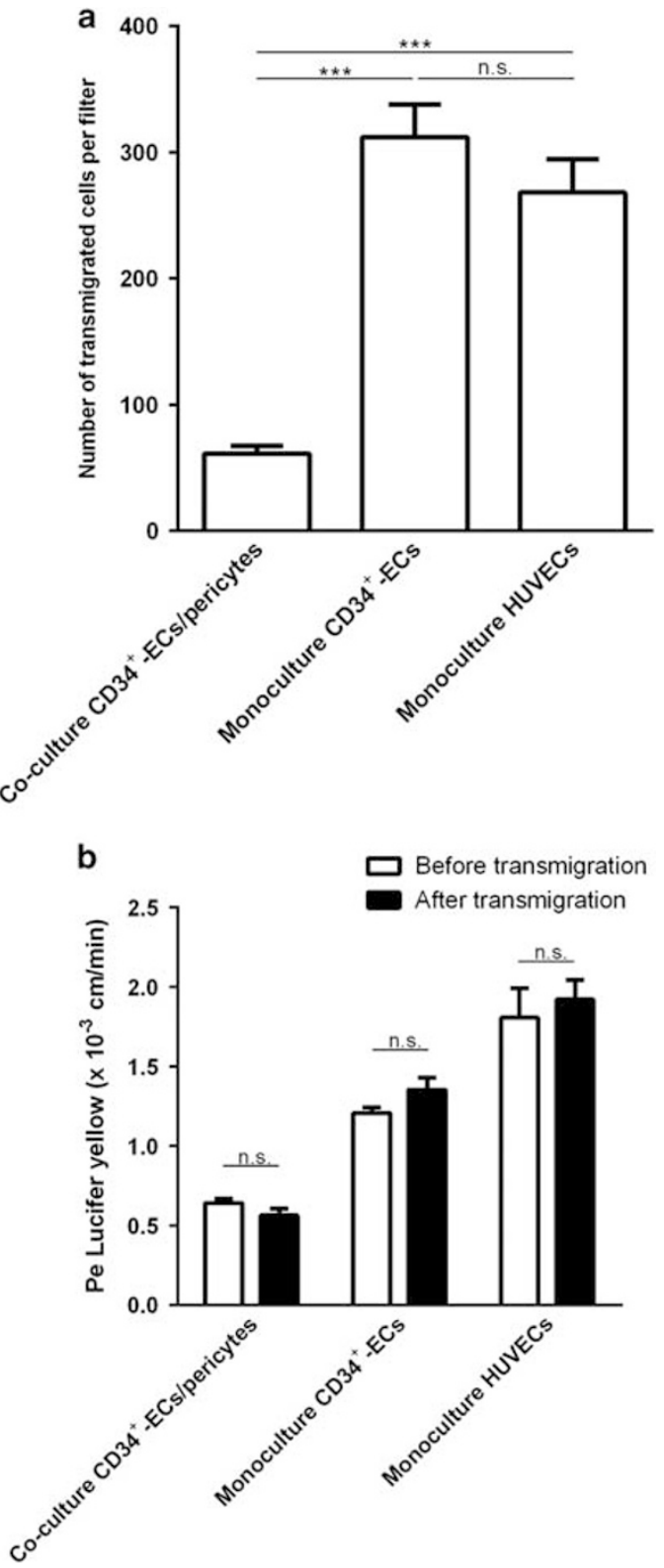

Figure 5 Transmigration of the MDA-MB-231 breast cancer cell line through our in vitro model of the human blood-brain barrier (BBB) and through endothelia with no BBB function: $\mathrm{CD}_{3} 4^{+}$-derived endothelial cells (ECs) and human umbilical vein ECs (HUVECs) in monoculture on 3- $\mu$ mpore inserts. (a) The number of transmigrated MDA-MB-231 cells on the lower face of cell culture inserts after $16 \mathrm{~h}$ (80 000 cancer cells were seeded in the upper compartment at the beginning of the experiment). The results are presented as the mean \pm s.e.m. of three experiments, each of which was performed in triplicate. For statistical analysis, an unpaired $t$-test was performed ${ }^{* * *} P<0.001 ;$ n.s., not significant). (b) Permeability of the endothelium to Lucifer yellow (LY) before and after a 16-h transmigration experiment. The results are presented as the mean \pm s.e.m. of three experiments, each of which was performed in triplicate. For statistical analysis, an unpaired $t$-test was performed (n.s., not significant). molecular events preceding brain metastasis formation in breast cancer and other tumors. Interestingly, the number of transmigrated MDA-MB-231 cells after $16 \mathrm{~h}$ was higher with endothelia with no BBB features (which actually exhibited a higher permeability to LY in our study), suggesting that they more easily cross-peripheral-like endothelia. It is important to note that triple-negative breast cancer patients commonly develop secondary tumors in peripheral organs (liver, bone, lung), ${ }^{31}$ beyond brain metastases that often arise later during the disease progression. ${ }^{32}$ For this reason, it has been suggested that breast cancer tumor cells need some time to develop their ability to penetrate through the $\mathrm{BBB}$ and colonize the brain. ${ }^{33}$ In this frame, it would be interesting to investigate and compare the ability of metastatic breast cancer cells to interact with the endothelium from different organs (adhesion, transmigration processes).

Different types of investigation require the growth of ECs on large-pore-size inserts (eg, studying the transport of large molecules such as antibodies and proteins across endothelial barriers). The technique herein described conserves a physiological, single endothelial monolayer on one face of the insert membrane. In principal, this simple but effective method could be easily implemented in cell culture laboratories using similar coculture systems. Moreover, the in vitro BBB model described here appears to be a useful tool for investigating cancer cell migration and may serve as a basis for further mechanistic and molecular studies in brain metastasis research.

\section{ACKNOWLEDGMENTS}

The authors wish to thank the Nord-Pas de Calais Regional Council for granting a postdoctoral fellowship to Elodie Vandenhaute and a PhD fellowship to Aurore Drolez. They also thank Béthune Hospital (Beuvry, France), and in particular Patrick Jacson (Chief Executive), Dr. Fabienne Viala (Head of the Maternity Unit), Jean-Yves Caudrelier (Senior Health Manager in Gynecology and Obstetrics), and the team of midwives for collecting cord blood samples. Lastly, authors are grateful to Professor Romeo Cecchelli for critically revising the manuscript. HUVECs were a king gift of Pr Lino Ferreira (University of Coimbra, Coimbra, Portugal).

\section{DISCLOSURE/CONFLICT OF INTEREST}

The authors declare no conflict of interest.

1. Reymond N, D'Água BB, Ridley AJ. Crossing the endothelial barrier during metastasis. Nat Rev Cancer [Internet]. Nature Publishing Group; December 2013 [cited 26 May 2014];13(12):858-870. Available at: http://www.ncbi. nlm.nih.gov/pubmed/24263189 [accessed 31 December 2015].

2. Zhou ZN, Boimel PJ, Segall JE. Tumor-stroma: in vivo assays and intravital imaging to study cell migration and metastasis. Drug Discov Today Dis Model 2011;8:95-102.

3. Bersini $S$, Jeon JS, Moretti $M$ et al. In vitro models of the metastatic cascade: from local invasion to extravasation. Drug Discov Today [Internet]. Elsevier Ltd; June 2014 [cited 16 June 2014];19(6): 735-742. Available at: http://www.ncbi.nlm.nih.gov/pubmed/ 24361339 [accessed 31 December 2015].

4. Samatov TR, Shkurnikov MU, Tonevitskaya SA et al. Modelling the metastatic cascade by in vitro microfluidic platforms. Prog Histochem Cytochem [Internet]. 7 February 2015 [cited 26 March 2015];49(4):2129. Available at: http://www.sciencedirect.com/science/article/pii/ S0079633615000029 [accessed 31 December 2015].

5. Pouliot Normand, Pearson, Helen B et al. Investigating metastasis using in vitro platforms. In: Jandial R, editor. Metastatic Cancer: Clinical and 
Biological Perspectives [Internet]. Landes Bioscience: Austin, TX, USA; 2000 [cited 30 June 2014], pp 77-100. Available at: http://www.ncbi. nlm.nih.gov/books/NBK100379/ [accessed 31 December 2015].

6. Paku S, Döme B, Tóth $\mathrm{R}$ et al. Organ-specificity of the extravasation process: an ultrastructural study. Clin Exp Metastasis 2000;18: 481-492.

7. Brastianos PK, Curry WT, Oh KS. Clinical discussion and review of the management of brain metastases. J Nat/ Compr Canc Netw [Internet]. 1 September 2013 [cited 14 July 2014];11(9):1153-1164. Available at: http://www.jnccn.org/content/11/9/1153.abstract.

8. Basu SK, Remick SC, Monga M et al. Breaking and entering into the CNS: clues from solid tumor and nonmalignant models with relevance to hematopoietic malignancies. Clin Exp Metastasis [Internet]. 2014 February [cited 14 July 2014];31(2):257-267. Available at: http://www. ncbi.nlm.nih.gov/pubmed/24306183.

9. Tóth A, Veszelka S, Nakagawa $S$ et al. Patented in vitro blood-brain barrier models in CNS drug discovery. Recent Pat CNS Drug Discov 2011;6:107-118.

10. Deli MA, Ábrahám CS, Kataoka $\mathrm{Y}$ et al. Permeability studies on in vitro blood-brain barrier models: physiology, pathology, and pharmacology. Cell Mol Neurobiol 2005;25:59-127.

11. Cecchelli R, Aday S, Sevin E et al. A stable and reproducible human blood-brain barrier model derived from hematopoietic stem cells. PLoS One [Internet]. 2014 January [cited 18 June 2014];9(6):e99733. Available at: http://www.pubmedcentral.nih.gov/articlerender.fcgi? artid $=$ 4061029\&tool $=$ pmcentrez\&rendertype $=$ abstract.

12. Lorger $\mathrm{M}$, Lee $\mathrm{H}$, Forsyth JS et al. Comparison of in vitro and in vivo approaches to studying brain colonization by breast cancer cells. J Neurooncol [Internet]. 2011 September [cited 16 Jun 2014];104 (3):689-696. Available at: http://www.ncbi.nlm.nih.gov/pubmed/ 21359851.

13. Pedroso DCS, Tellechea A, Moura L et al. Improved survival, vascular differentiation and wound healing potential of stem cells co-cultured with endothelial cells. PLoS One [Internet]. 2011 January [cited 30 May 2014];6(1):e16114. Available at: http://www.pubmedcentral.nih.gov/ articlerender.fcgi?artid $=3026015 \&$ tool $=$ pmcentrez\&rendertype $=$ abstract.

14. Röhnelt RK, Hoch G, Reiß Y et al. Immunosurveillance modelled in vitro: naive and memory $\mathrm{T}$ cells spontaneously migrate across unstimulated microvascular endothelium. Int Immunol 1997;9:435-450.

15. Cecchelli R, Dehouck B, Descamps $L$ et al. In vitro model for evaluating drug transport across the blood-brain barrier. Adv Drug Deliv Rev 1999;36:165-178.

16. Czupalla CJ, Liebner S, Devraj K. In vitro models of the blood-brain barrier. Methods Mol Biol [Internet]. 2014;1135:415-437. Available at: http://www.ncbi.nlm.nih.gov/pubmed/24510883 [accessed 31 December 2015].

17. Eigenmann D, Xue G, Kim K et al. Comparative study of four immortalized human brain capillary endothelial cell lines, hCMEC/D3, hBMEC, TY10, and BB19, and optimization of culture conditions, for an in vitro blood-brain barrier model for drug permeability studies. Fluids Barriers CNS [Internet]. 2013;10(1):33. Available at: http://www. fluidsbarrierscns.com/content/10/1/33 [accessed 31 December 2015].

18. Tilling $\mathrm{T}$, Engelbertz $\mathrm{C}$, Decker $\mathrm{S}$ et al. Expression and adhesive properties of basement membrane proteins in cerebral capillary endothelial cell cultures. Cell Tissue Res 2002;310:19-29.
19. Engler C, Kelliher C, Speck CL et al. Assessment of attachment factors for primary cultured human corneal endothelial cells. Cornea [Internet]. 2009;28:1050-4. Available at: http://www.ncbi.nlm.nih.gov/pubmed/ 19724204 [accessed 31 December 2015].

20. Dohgu S, Takata F, Yamauchi A et al. Brain pericytes contribute to the induction and up-regulation of blood-brain barrier functions through transforming growth factor- $\beta$ production. Brain Res 2005;1038: 208-215.

21. Thanabalasundaram G, Schneidewind J, Pieper C et al. The impact of pericytes on the blood-brain barrier integrity depends critically on the pericyte differentiation stage. Int J Biochem Cell Biol 2011;43: 1284-1293.

22. Al Ahmad A, Taboada CB, Gassmann M et al. Astrocytes and pericytes differentially modulate blood-brain barrier characteristics during development and hypoxic insult. J Cereb Blood Flow Metab 2011;31: 693-705.

23. Nakagawa S, Deli MA, Nakao $S$ et al. Pericytes from brain microvessels strengthen the barrier integrity in primary cultures of rat brain endothelial cells. Cell Mol Neurobiol 2007;27:687-694.

24. Daneman R, Zhou L, Kebede AA et al. Pericytes are required for bloodbrain barrier integrity during embryogenesis. Nature 2010;468:562-566.

25. Armulik A, Genové G, Mäe $M$ et al. Pericytes regulate the blood-brain barrier. Nature [Internet]. 25 November 2010 [cited 10 July 2014];468 (7323):557-561. Available at: http://www.ncbi.nlm.nih.gov/pubmed/ 20944627 [accessed 31 December 2015].

26. Liebner S, Plate KH. Differentiation of the brain vasculature: the answer came blowing by the Wnt. J Angiogenes Res 2010;2:1.

27. Alvarez Jl, Katayama T, Prat A. Glial influence on the blood-brain barrier. Glia 2013;61:1939-1958.

28. Nguyen DX, Bos PD, Massagué J. Metastasis: from dissemination to organ-specific colonization. Nat Rev Cancer 2009;9:274-284.

29. Hambrecht A, Jandial R, Neman J. Emerging role of brain metastases in the prognosis of breast cancer patients. Breast Cancer (Dove Med Press [Internet]. 2011;3:79-91. Available at: http://www.pubmedcentral.nih.gov/ articlerender.fcgi?artid $=3846823 \&$ tool $=$ pmcentrez\&rendertype $=$ abstract [accessed 31 December 2015].

30. Rodriguez $\mathrm{PL}$, Jiang $\mathrm{S}, \mathrm{Fu} \mathrm{Y}$ et al. The proinflammatory peptide substance $P$ promotes blood-brain barrier breaching by breast cancer cells through changes in microvascular endothelial cell tight junctions. Int J Cancer [Internet]. 1 March 2014 [cited 16 June 2014];134(5):103444. Available at: http://www.ncbi.nlm.nih.gov/pubmed/23934616 [accessed 31 December 2015].

31. Kimbung S, Loman N, Hedenfalk I. Clinical and molecular complexity of breast cancer metastases. Semin Cancer Biol [Internet]. 2015 August [cited 29 August 2015];35:85-95. Available at: http://www. sciencedirect.com/science/article/pii/S1044579X15000723 [accessed 31 December 2015].

32. Leone JP, Lee A V, Brufsky AM. Prognostic factors and survival of patients with brainmetastasis from breast cancer who underwent craniotomy. Cancer Med [Internet]. Wiley: Chichester, UK; 9 July 2015;4 (7):989-994. Available at: http://www.ncbi.nlm.nih.gov/pmc/articles/ PMC4529337/ [accessed 31 December 2015].

33. Leone JP, Leone BA. Breast cancer brain metastases: the last frontier. Exp Hematol Oncol [Internet]. BioMed Central: London; 24 November 2015;4:33. Available at: http://www.ncbi.nlm.nih.gov/pmc/articles/ PMC4657380/ [accessed 31 December 2015]. 